\title{
1 Sensitive, reliable, and robust circRNA detection from RNA-seq with
}

\section{CirComPara2}

3 Enrico Gaffo ${ }^{1, *}$, Alessia Buratin ${ }^{1,2}$, Anna Dal Molin ${ }^{1}$, Stefania Bortoluzzi ${ }^{1,3, *}$

$4{ }^{1}$ Department of Molecular Medicine, University of Padova, Padova, Italy

$5 \quad 2$ Department of Biology, University of Padova, Padova, Italy

$6{ }^{3}$ Interdepartmental Center For Innovative Biotechnologies, University of Padova, Padova, Italy

$7 \quad$ * corresponding authors

8 Tel: +39-049-827-6502

9 Fax: +39-049-827-6208

10 Email: stefania.bortoluzzi@unipd.it (S.B.), enrico.gaffo@unipd.it (E.G.)

11 Running title: CircRNA detection from RNA-seq with CirComPara2

12 Keywords: circRNAs, bioinformatics, transcriptomics, RNA-seq 


\section{Abstract}

14 Circular RNAs (circRNAs) are a large class of covalently closed RNA molecules that originate by a

15 process called back-splicing. CircRNAs are emerging as functional RNAs involved in the regulation

16 of biological processes as well as in disease and cancer mechanisms. Current computational methods

17 for circRNA identification from RNA-seq experiments are characterised by low discovery rates and

18 performance dependent on the analysed data set. We developed a new automated computational

19 pipeline, CirComPara2 (https://github.com/egaffo/CirComPara2), that consistently achieves high

20 recall rates without losing precision by combining multiple circRNA detection methods. In our

21 benchmark analysis, CirComPara2 outperformed state-of-the-art circRNA discovery tools and proved

22 to be a reliable and robust method for comprehensive transcriptomics characterisation.

\section{Introduction}

24 Recent research uncovered that eukaryotic transcriptomes comprise thousands of stable circular RNAs

25 (circRNAs) that originate by a process called back-splicing, where the transcript 3' and 5' ends are covalently joined (Xiao et al. 2020). Rather than being transcriptional byproducts, circRNA molecules exert critical functions in cell biology through different mechanisms (Bonizzato et al. 2016). By interacting with microRNAs, circRNAs can regulate gene expression and govern important oncogenic axes (Hansen et al. 2013); moreover, similarly to long non-coding RNAs, they can control diverse cellular processes by decoying RNA-binding proteins and scaffolding molecular complexes (Du et al.

31 2016). CircRNAs can also function as templates for translation to encode functional peptides (Xiao et al. 2020)(Wu et al. 2021b) and regulate the transcription of their parental gene (Li et al. 2015).

33 Nowadays, circRNAs are considered key players that can impact all the cancer hallmarks (Hanniford 34 et al. 2020; Slack and Chinnaiyan 2019). The discovery of circRNA regulatory roles and their 35 potential as biomarkers given by higher stability compared to linear RNAs (Rajappa et al. 2020) 36 have actuated the integration of circRNA investigation in conventional transcriptomics, especially in cancer research and studies of pathological conditions (Santer et al. 2019; Hua et al. 2019), including 
viral infections (Awan et al. 2021).

39 Studies of circRNAs rapidly increased in pace thanks to the development of bioinformatics tools that

40 identify the sequences spanning circRNA back-splice junctions from total RNA-seq data. To date,

41 several methods for circRNA identification have been developed (Chen et al. 2020). Still, none

42 outperforms the others since they all provide either highly sensitive or highly precise predictions and

43 highly variable performance across benchmark data sets (Chen et al. 2015; Hansen et al. 2016; Zeng

44 et al. 2017). Interestingly, Hansen (Hansen 2018) observed that circRNA detection methods largely

45 agreed on true predictions, whereas circRNAs identified by single methods were enriched in false-

46 positive guesses (FPs) and suggested selecting circRNAs commonly predicted by two or more

47 methods to obtain dependable results.

48 We formerly implemented CirComPara (Gaffo et al. 2017), an automated computational pipeline

49 combining four circRNA detection methods, including CIRCexplorer (Zhang et al. 2014), CIRI2 (Gao

50 et al. 2018), Findcirc (Memczak et al. 2013) and Segemehl (Hoffmann et al. 2014). CirComPara

51 controlled the FP number by considering only the circRNAs commonly detected by two or more

52 methods.

53 In CirComPara2, we have considerably improved our tool by: (i) including five additional circRNA

54 detection methods, (ii) updating the software of the already integrated tools, (iii) implementing a more

55 accurate counting of the back-spliced reads, (iv) increasing the analysis pipeline flexibility, and (v)

56 including the procedure to calculate the linear expression related to the circRNAs.

57 In this work, we first show that nine widely used circRNA detection tools could miss circRNAs of

58 interest. Then, we confirm that CirComPara2 correctly reports circRNAs overlooked by other

59 methods and achieves significantly higher sensitivity with no loss of precision. Finally, assessment on

60 simulated data and 142 public RNA-seq samples demonstrated the consistent higher performance of

61 CirComPara2 compared with state-of-the-art methods. 


\section{Results}

63 CircRNA detection methods could miss abundant circRNAs.

64 We simulated RNA-seq expression data of 5,680 circRNAs from the whole human genome

65 ("simulated data set"; see Methods) to evaluate the characteristics of circRNA detection methods'

66 false-negative predictions (FNs), i.e., true circRNAs not identified as such. We applied nine widely

67 used computational pipelines for circRNA discovery including circRNA_finder (Westholm et al.

68 2014), CIRI2 (Gao et al. 2018), DCC (Cheng et al. 2016), Findcirc (Memczak et al. 2013), Segemehl

69 (Hoffmann et al. 2014), and CIRCexplorer2 (Zhang et al. 2016). CIRCexplorer2 was coupled to any

70 of BWA (Li 2013) (C2BW), Segemehl (C2SE), STAR (Dobin et al. 2013) (C2ST) and TopHat-

71 Fusion (Kim and Salzberg 2011) (C2TH) aligners, thus composing four different pipelines.

72 On average, $49 \%$ of FNs detected by each method showed higher expression than the overall

73 circRNA median expression (Figure 1a), suggesting that nearly half of the missed circRNAs had a

74 considerable expression level no matter which method was applied. Besides, the expression

75 distribution of the FNs was similar to the correctly identified circRNAs, i.e., the true-positive findings

76 (TPs), whereas the false-positive (FP) expression was generally low. 
bioRxiv preprint doi: https://doi.org/10.1101/2021.02 18.431705; this version posted May 21, 2021. The copyright holder for this preprint (which was not certified by peer review) is the author/funder, who has granted bioRxiv a license to display the preprint in perpetuity. It is made available under aCC-BY-NC-ND 4.0 International license.
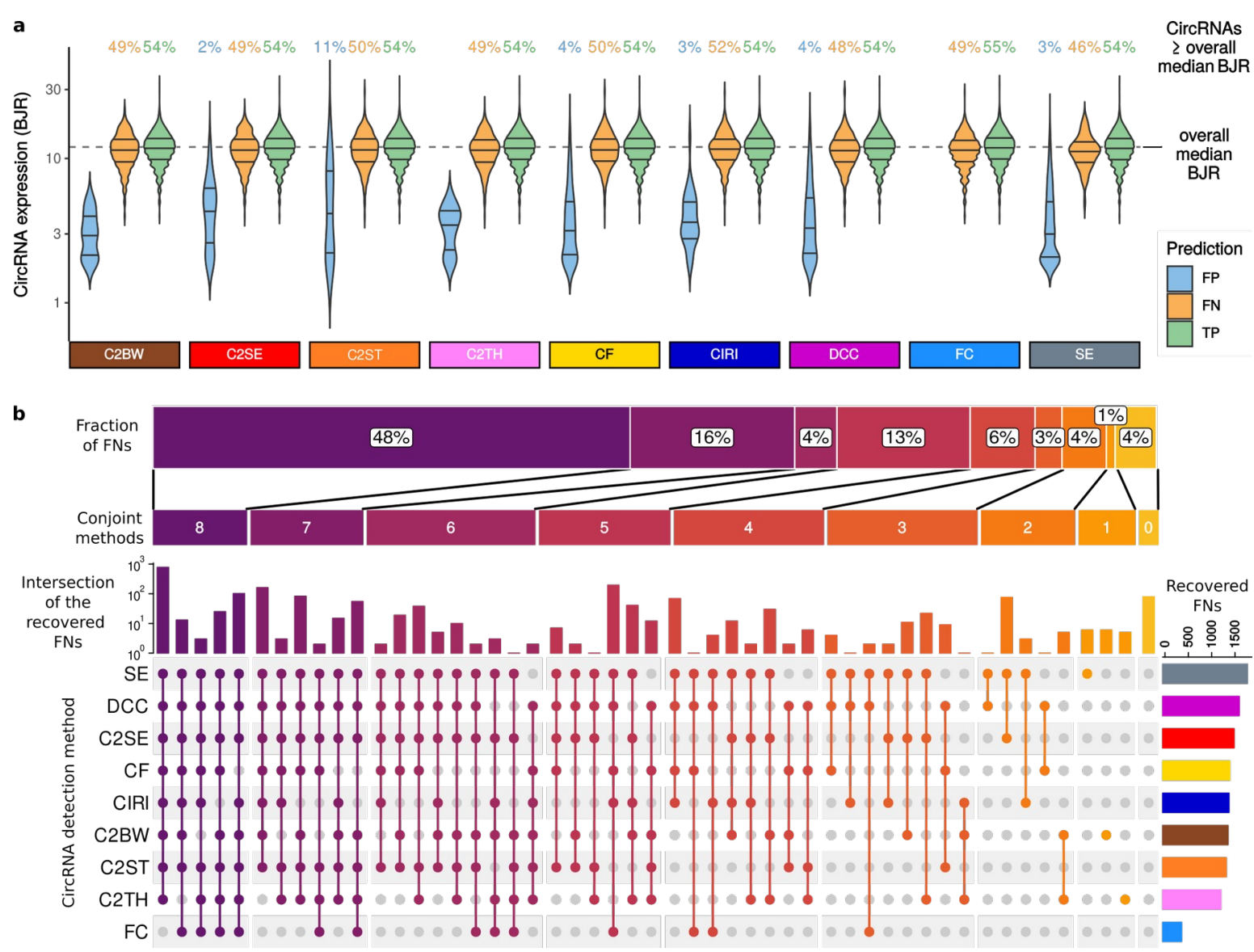

Figure 1. CircRNAs overlooked by common detection methods and the CirComPara2 approach. a, The expression level of predicted circRNAs. BJR: back-splice junction read counts; C2BW: CIRCexplorer2 on BWA; C2SE: CIRCexplorer2 on Segemehl; C2ST: CIRCexplorer2 on STAR; C2TH: CIRCexplorer2 on TopHat-Fusion; CF: circRNA_finder; CIRI: CIRI2; FC: Findcirc; SE: Segemehl; FP: false-positive; FN: false negative; TP: true positive. b, Number of methods detecting circRNAs not detected by other methods and the number of the FNs detected. Colour refers to the number of methods conjointly detecting circRNAs missed by other tools. The vertical bars show the number of FN circRNAs detected by the methods indicated in the coloured dots below the bars. The bars denote disjoint circRNA sets. Grey dots indicate the methods failing to detect the circRNAs considered in the bar chart on the top. The horizontal bars on the right represent the overall number of FN circRNAs detected by the methods. The horizontal bar shows the percentage of detected FNs by grouping method combinations according to the number of combined methods.

Multiple methods compensate each other, and method combinations increase the detection sensitivity.

We further examined the 1,945 circRNAs undetected by one or more tools, referred to as the "FN set" from now on, by counting how many circRNAs in the FN set each method could detect.

Interestingly, only $4 \%$ circRNAs of the FN set were undetected by all methods (Figure 1b), whereas 
$8296 \%$ could be identified at least by one among the nine tools. Specifically, 1\% FNs were detected

83 individually by Segemehl, $\mathrm{C} 2 \mathrm{BW}$, and $\mathrm{C} 2 \mathrm{TH}$; and $95 \%$ were commonly identified by various

84 combinations of two or more tools. Almost half the FNs (48\%) were conjointly detected by eight out

85 of nine methods, with Segemehl, DCC and C2SE providing the most inclusive predictions. Instead,

86 Findcirc showed the least number of recovered FNs. However, no method entirely covered the

87 predictions of Findcirc, indicating some specificity of its algorithm.

88 Overall, this analysis suggested that algorithms with possibly different and complementary features

89 can compensate each other and improve the detection rate if applied together.

\section{Workflow and features of CirComPara2}

91 Following the observation reported in the previous paragraph, we enhanced CirComPara by including

92 more circRNA detection methods to improve its sensitivity. Moreover, we introduced new features

93 that made CirComPara2 more flexible, computationally efficient, and resilient.

94 CirComPara2 implements a fully automated computational pipeline for circRNA detection,

95 quantification, annotation, and integration with linear gene expression data (Figure 2a). Several

96 parameters are available to customise the analysis workflow and the integrated methods. The minimal

97 input consists of the RNA-seq reads in FASTQ format and a reference genome in FASTA format.

98 Optionally, the user can also provide the gene annotation in GTF format. The software will then build

99 the genome indexes for each read aligner and perform the necessary file format conversions.

100 Previously computed indexes can be reused as input to save computing time. 


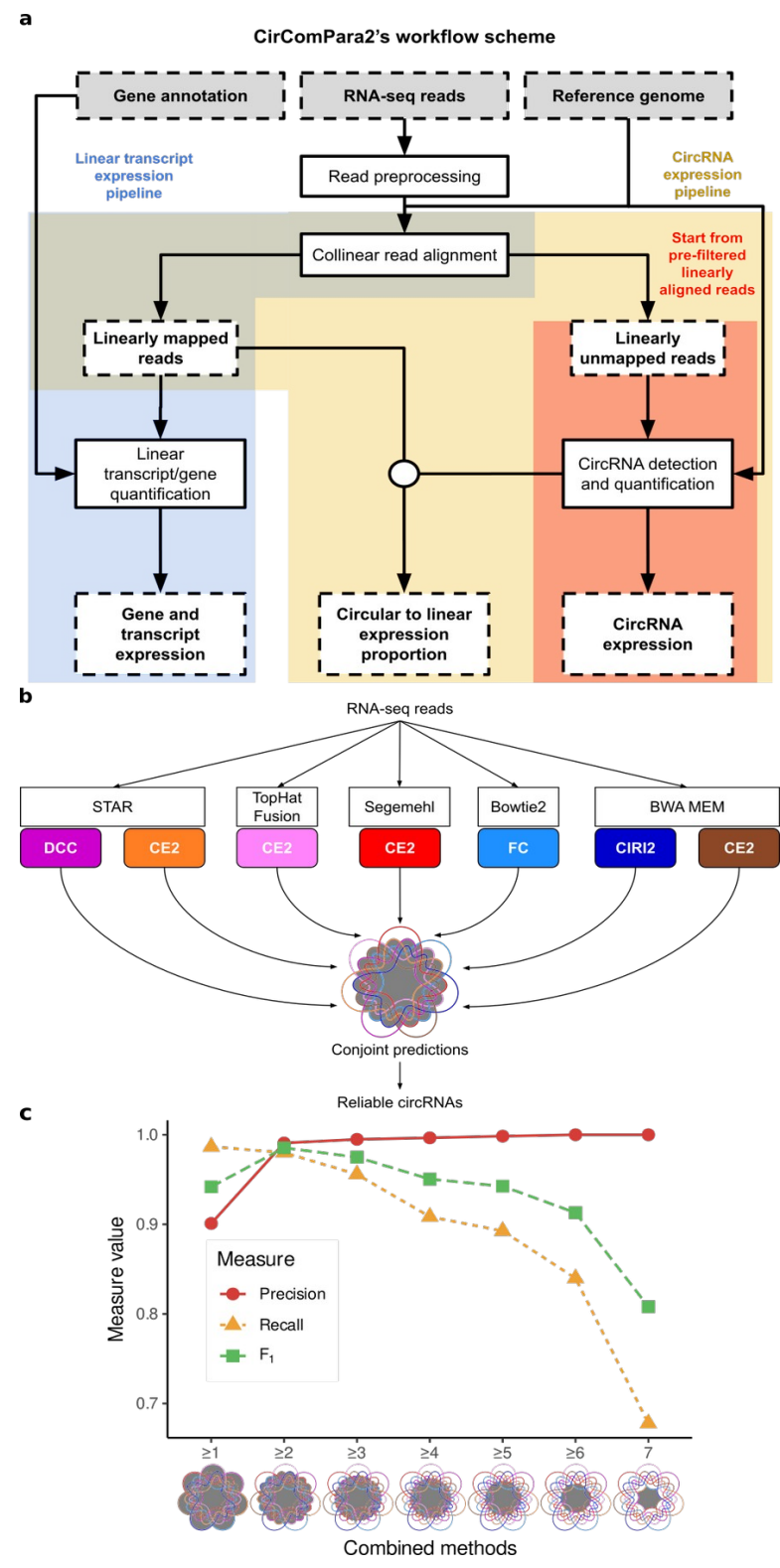

Figure 2. The CirComPara2 workflow and approach. a,

Boxes with dashed contour indicate input and output data; boxes with solid-line contour indicate computing tasks; the circular connector indicates merging data. Background colours highlight the different pipeline branches (linear transcript analysis in blue, full circRNA analysis in yellow, and strict circRNA analysis in red). b, Detail of the CirComPara2 strategy ("CircRNA detection and quantification" box in a) showing the embedded circRNA detection methods (coloured rounded corner boxes) with the respective chimeric read aligners (white boxes). The central Venn diagram represents the optimised combination of method prediction intersections implemented by CirComPara2 with circRNAs conjointly detected by two or more methods (grey filled intersections) retained as default. Method abbreviations as in Figure 1. c, Precision (red), recall (yellow) and $F_{1}$-score (green) for different numbers of methods considered by CirComPara2: $\geq 1$ indicates the union of all single methods predictions, $\geq 2$ indicates any pair of methods, and so on, up to 7 , which indicates the conjoint predictions of all the methods considered. The grey filled parts in Venn diagrams show the intersections considered by the conjoint method combinations.

101 The CirComPara2 workflow comprises an optional pre-processing of the input raw reads by

102 Trimmomatic (Bolger et al. 2014) to trim or discard low-quality reads. Statistics of the preprocessing

103 steps are produced with the FastQC tool (Andrews 2010). Next, the reads are aligned collinearly to

104 the reference genome using HISAT2 (Kim et al. 2019) to (i) identify the reads that are later used for

105 linear transcript analysis (Figure 2a) and (ii) extract the reads not collinearly aligned, which are used

106 to detect back-splices. The linear gene and transcript expression analysis is performed with StringTie

107 (Pertea et al. 2015) and produces files that can be easily imported into packages for downstream

108 expression analysis such as tximport (Soneson et al. 2015) and tximeta (Love et al. 2020). The 
108

109

110

111

112

113

114

115

116

117

118

119

120

121

122

123

124

125

126

127

128

129

130

131

132

133

134

circRNA analysis aligns the collinearly unmapped reads independently with five methods allowing chimeric alignments, namely Bowtie2 (Langmead and Salzberg 2012), BWA-MEM, Segemehl, STAR and TopHat-Fusion (Figure 2b). The chimeric aligner outputs are subsequently parsed by six circRNA detection tools, which compose the nine different circRNA detection sub-pipelines presented in the previous paragraph. Of note, the computationally expensive chimeric alignment step is performed only once per aligner and reused by multiple circRNA detection tools, boosting CirComPara2 efficiency. For instance, the same alignments from STAR are passed to CIRCexplorer2, circRNA_finder, and DCC (Figure 2b). The outputs of the various tools are automatically handled, converting them into a standard format to compare the predicted back-splices. Moreover, the identifiers of the back-spliced reads are collected while keeping track of the predicting method to obtain non-redundant read counts for each circRNA. Finally, the linear expression of circRNA host genes is evaluated by counting the reads collinearly mapped at each back-splice junction using bedtools (Quinlan and Hall 2010), GNU parallel (Tange 2020), and custom scripts.

CirComPara2 has a modular and highly parallelised implementation that makes it computationally efficient and resilient. By using custom parameters, CirComPara2 allows skipping computation tasks that are not of interest to the user. For instance, the user can select to run only the pipeline branch computing the linear or the circular transcript analysis, the collinear alignments (for instance, if they were previously computed), or both the collinear alignment and the linear transcript pipeline branch, therefore performing only the circRNA detection from pre-filtered reads. Plus, the Scons (www.scons.org) engine is leveraged to run independent tasks in parallel, resume an interrupted analysis by performing only uncompleted tasks, and, if the user changed some parameters from a previous run, compute only the tasks dependent on the modified parameters.

CirComPara2 is available as standalone software (https://github.com/egaffo/circompara2) and Docker image (https://hub.docker.com/r/egaffo/circompara2), which facilitates installation, portability, and reproducibility of the analysis.

\section{Optimal method combination for circRNA detection}

Concurrently to the improvement of CirComPara2 sensitivity, we wanted to control the number of the 
135 introduced FPs to preserve a high precision. As observed in a previous study (Hansen 2018), the

136 combination of specific methods does not ameliorate the discovery of true circRNAs. Accordingly,

137 our method combination assessment on the simulated data showed that combining circRNA_finder or

138 Segemehl with other methods contributed to increasing the FP number (Supplementary Figure S1).

139 For this reason, circRNA_finder and Segemehl were excluded in the CirComPara2 default method

140 combination (Figure 2b); nevertheless, these two methods can be included if enabled by the user.

141 Next, we fine-tuned the number of conjoint methods considering the amount of recovered FNs against

142 the introduced FPs to optimise the method combination strategy. As expected, considering the

143 predictions from all methods resulted in the highest recall (0.99) and the lowest precision (0.90)

144 among the combination strategies (Figure 2c). Further, excluding predictions from single methods,

145 i.e., selecting only the circRNAs commonly detected by two or more tools, showed a slightly reduced

146 recall $(0.98)$ with a substantially increased precision $(0.99)$. This large precision gain indicated that

147 most of the FPs were predicted by single methods. Further increasing the number of conjoint methods

148 (i.e., from three-or-more to all-seven methods conjoint) lead to a considerable decrease of recall (0.96

149 to 0.68$)$ with only a modest increase of precision ( 0.99 to 1.00$)$.

150 To evenly weigh recall and precision in ranking the method combination strategies, we calculated the

$151 \mathrm{~F}_{1}$-score for each combination. The best trade-off between recall and precision, indicated by the

152 highest $\mathrm{F}_{1}$-score (0.99), was obtained with the two-or-more method strategy (Figure 2c).

\section{CirComPara2 outperforms other methods in simulated data.}

154 We next set CirComPara2 with the two-or-more method strategy and compared it with the single

155 methods on the simulated data. CirComPara2 obtained the highest $\mathrm{F}_{1}$-score (0.99) by achieving the

156 highest recall (0.98) while holding a precision comparable to or higher than the other algorithms $(0.99$

157 versus 0.92-1.00; Figure 3a), confirming that CirComPara2 rectified the true circRNAs missed by the

158 other methods. 

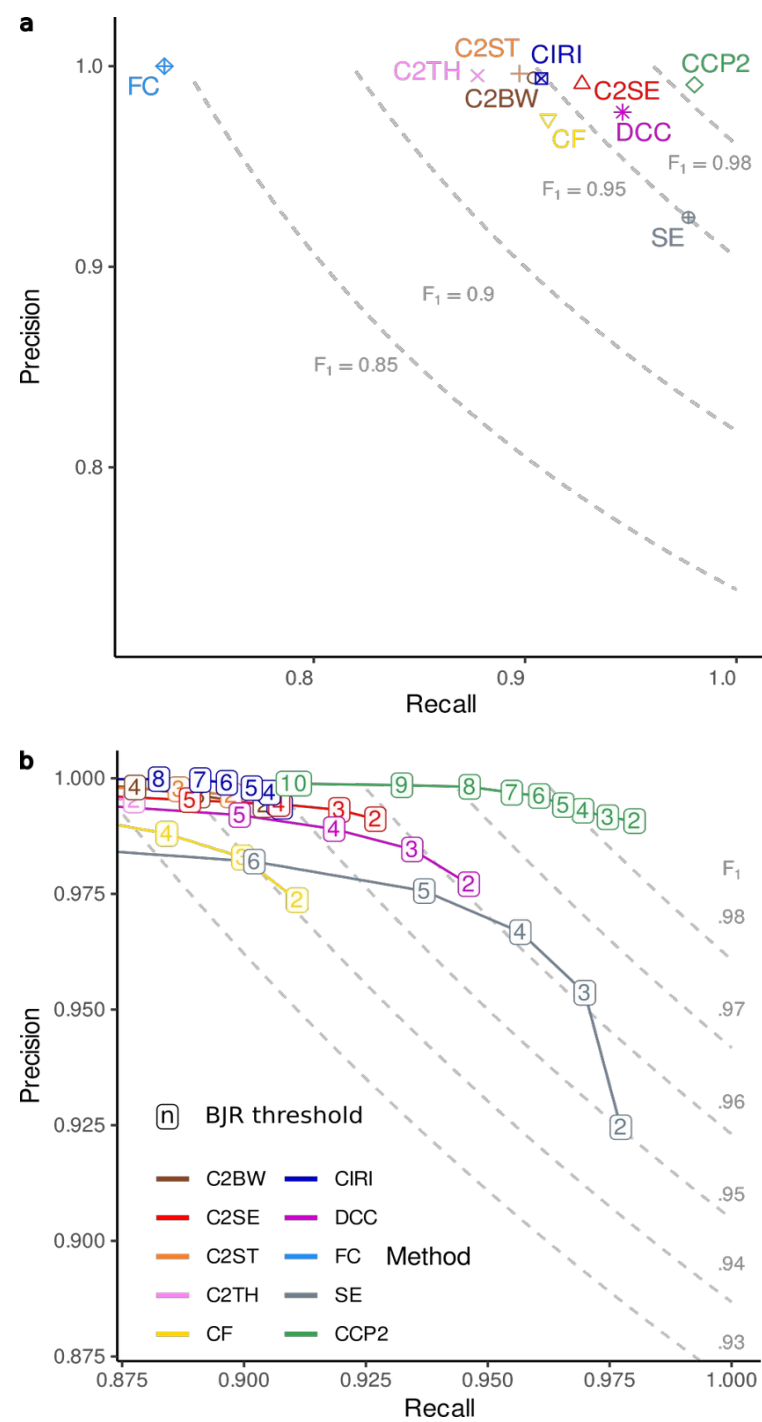

Figure 3. Performance of circRNA detection methods on the simulated data set. a, Precision and recall of the circRNA detection methods (labels as in Figure 1), including CirComPara2 (CCP2; green dot); the dashed-line curves display plot areas for $0.85,0.90,0.95$ and $0.98 \mathrm{~F}_{1^{-}}$ scores. b, CircRNA detection methods' performance upon application of filters from 2 to 10 minimum circRNA raw expression estimates (BJR: back-splice junction read counts); dashed-line curves delimit $\mathrm{F}_{1}$-scores plot areas from 0.92 to 0.98 with 0.01 increase steps; method colours as in (a).

159 To assess the extent of the annotation-guided method contribution to CirComPara2 predictions, we

160 performed the analysis also with a pruned gene annotation input to the algorithms (see Methods). As

161 expected, the CIRCexplorer2 pipelines showed a dramatic reduction (up to -0.14) of the recall and $\mathrm{F}_{1}$ scores (Supplementary Figure S2). Instead, CirComPara2 maintained the highest $\mathrm{F}_{1}$-score (0.98), suggesting that it can be efficient when applied to RNA-seq data of organisms with incomplete or poor genome annotation by leveraging the embedded annotation-independent tools.

A typical circRNA expression analysis usually involves post-detection data cleaning to remove background noise signals or mapping errors and poorly expressed circRNAs of little interest (Chen et al. 2016). Consequently, the circRNAs with small back-splice junction read counts (BJRs) are routinely filtered out. As shown in Figure 1a, FPs generally have small BJRs, suggesting that more reliable circRNAs can be retained by simply filtering according to expression abundance. We applied 
this procedure to our data, progressively filtering out circRNAs with less than two up to $10 \mathrm{BJRs}$. We stress that removing circRNAs with $\leq 10$ BJRs was an extreme filter since it was close to the overall median BJR (Figure 1a). As expected, by increasing the minimum-BJR filter threshold, all methods scored higher precision but with a corresponding reduced recall, indicating that true circRNAs were discarded as well (Supplementary Figure S3). Nevertheless, CirComPara2 maintained the highest recall compared to other methods at equal precision (Figure 2b; Supplementary Table S1), suggesting that the circRNAs recovered by CirComPara2 were valid findings with a considerable abundance that may represent relevant circRNAs in actual experiments.

\section{Benchmark real data sets}

The gold standard for evaluating circRNA detection methods on real RNA-seq data is to compare ribosomal RNA-depleted (ribo-) with circRNA-enriched sequencing libraries. The most used technique is the additional treatment of the ribo- library with RNase R to exploit the exonuclease degradation resistance of circRNAs derived by their lack of a single-stranded $3^{\prime}$ end (Hansen et al. 2016; Hansen 2018; Gao et al. 2018; Zeng et al. 2017; Szabo and Salzman 2016). Accordingly, we collected a total of 142 public real RNA-seq data samples for which sample-matched ribo ${ }^{-}$and RNase R-treated libraries were available (Table 1). This validation data set comprised samples of human cell lines and various human, Rhesus macaque, and mice tissues from six independent studies.

The matched RNase R-treated samples were used as a control to define the TP circRNAs (see Methods). Assuming that the RNase R treatment would deplete the linear more than the circular transcripts, we considered TPs the circRNAs having circular-to-linear expression proportion (CLP) higher or equal in the RNase R-treated compared to the ribo- matched samples according to at least one method. In this way, we accounted for different sequencing library depths between the matched samples. Moreover, each circRNA host-gene linear expression was estimated commonly to all the detection tools and independently of the circRNA abundance estimated by each method, allowing to remove possible advantages given by specific method quantification approaches. 


\begin{tabular}{ccc} 
SRA/GEO/BIGD ID & Tissue(s)/cell line(s) & Study reference \\
\hline $\begin{array}{c}\text { SRR3476956, SRR1636985, SRR1636986; } \\
\text { SRR3476958, SRR1637089, SRR1637090. } \\
\text { SRR3479244, SRR3479243 }\end{array}$ & HeLa, HEK293 & $\begin{array}{c}\text { (Gao et al. 2018) } \\
\text { (Gao et al. 2016) }\end{array}$ \\
\hline GSE130905 (10 samples) & HeLa & (Xiao and Wilusz 2019)* \\
\hline CRA001838 (8 samples) & HeLa & (Zhang et al. 2020) \\
\hline SRR444655, SRR444975; SRR445016, SRR444974 & Hs68 & (Jeck et al. 2013) \\
\hline GSE113120 (24 samples) & $\begin{array}{c}\text { 22Rv1, 42D, PC3, } \\
\text { V16, LNCaP }\end{array}$ & (Chen et al. 2019) \\
\hline PRJCA000751 (88 samples) & $\begin{array}{c}\text { Homo sapiens } \\
\text { (17 tissues) }\end{array}$ & \\
\cline { 2 - 3 } & Mus musculus & \\
(14 tissues) & \\
& Macaca mulatta & \\
& (13 tissues) & \\
\hline
\end{tabular}

Table 1. Real data sets of rRNA- and rRNA-/RNase R treated matched pairs of samples used to benchmark the circRNA detection methods. Tissues and cell lines are from humans unless specified.

* Data set GSE130905 used an improved protocol for circRNA enrichment (Xiao and Wilusz 2019). GEO:

Gene Expression Omnibus (Barrett et al. 2013), BIGD NGDC: National Genomics Data Center (National

Genomics Data Center Members and Partners 2020)

\section{CirComPara2 performs consistently better than single methods}

The performance of the methods on the real RNA-seq data agreed with the analysis on simulated data and previous benchmark study results (Zeng et al. 2017; Hansen 2018). Segemehl showed high recall (median 0.75; Figure 4a) but the lowest precision (median 0.92; Figure 4b), whereas C2BW, as expected from annotation-based algorithms, showed the most reliable predictions (median precision 0.97; Figure $4 \mathrm{~b}$ ). Similar to the evaluation on the simulated data set, we computed the $\mathrm{F}_{1}$-scores on the real data set. According to $\mathrm{F}_{1}$-score medians, CirComPara2 had the highest (0.91) and significantly different value $(\mathrm{q}<0.001$; Figure $4 \mathrm{c})$, and substantially outperformed the next best method, Segemehl (median $\mathrm{F}_{1}$-score 0.82 ), with a $0.09 \mathrm{~F}_{1}$-score difference (Supplementary Table S2). The highest $\mathrm{F}_{1}$ score, achieved by CirComPara2, resulted from a significantly larger recall (median 0.86; $\mathrm{q}<0.001$; Figure $4 \mathrm{a})$ and a negligible loss of precision compared to the other methods ( 0.01 median reduction to the most precise method; Figure 4b). Moreover, CirComPara2 had the narrowest interquartile range of $\mathrm{F}_{1}$-scores across the real-data sets $(0.11$; Figure $4 \mathrm{c}$; Supplementary Table $\mathrm{S} 2)$, proving that it is robust and almost unaffected by the experimental scenario. In light of the more challenging nature of real 
215 confirmed the advantage of CirComPara2 over the methods here assessed.

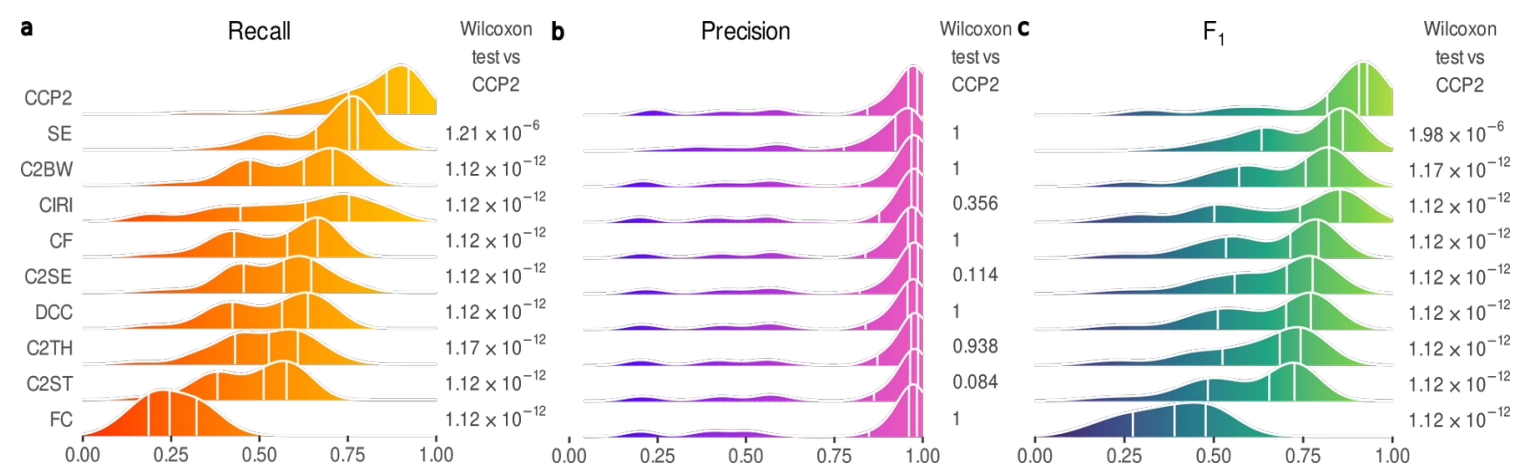

Figure 4. Performance of circRNA detection methods on the real RNA-seq data set. Results of the analysis on the 71 pairs of samples of the real RNA-seq data set. Density plot of (c) recall, (d) precision and (e) $\mathrm{F}_{1}$-score; distribution quartiles are indicated by white vertical lines. On the right-hand side of each plot, the Bonferroni adjusted p-values of Wilcoxon paired test contrasting each method with CirComPara2. Methods' abbreviations as in Figure 1; CCP2: CirComPara2.

\section{Discussion}

217 In the early days after circRNA discovery, bioinformaticians put significant effort into developing 218 circRNA detection methods with highly precise predictions. To this aim, the current circRNA 219 detection algorithms apply filtering procedures to remove FPs, but that may also reject true circRNAs 220 (Zeng et al. 2017; Chen et al. 2015; Hansen 2018; Hansen et al. 2016), increasing the number of FNs 221 and the risk of overlooking circRNAs of interest, as suggested by our analysis. Moreover, the frequent 222 practice of filtering out low count circRNAs to improve precision may result in the loss of 223 differentially expressed elements in comparative studies, as demonstrated by systematic studies on 224 gene expression (Zhu et al. 2019)(Buratin et al. 2020). Notably, unlike for FPs, experimental 225 validations cannot amend the bias derived by FN errors. These considerations prompted us to consider 226 a method evaluation metric that equally weighted precision and recall, such as the $\mathrm{F}_{1}$-score.

227 Interestingly, the evaluation by $\mathrm{F}_{1}$-score in our simulated data analysis highlighted that some methods 228 with diverging precision and recall, such as CIRI2 and Segemehl (Figure 3a), were equally ranked. Moreover, the real data set analysis showed an ample recall variation of each method across the 
231 confirmed that choosing a single method for circRNA discovery is problematic since no single best

232 performing method exists. We showed that an approach leveraging the advantages brought by each of

233 the integrated methods allows CirComPara2 to perform better than the single methods consistently in

234 different experimental contexts. Besides, we showed that CirComPara2 predictions are more inclusive

235 and robust than single method ones, even upon low count filtering, indicating that the recovered

236 circRNAs missed by other methods are not merely of low abundance.

237 To our knowledge, CirComPara has been the first bioinformatics tool to combine multiple circRNA

238 detection methods in an automated software pipeline. Since its former implementation, the continuous

239 upgrade of CirComPara evolved into a substantially improved new tool, CirComPara2, with an

240 efficacious method combination strategy. Other computational pipelines that employed multiple

241 circRNA detection tools, such as RAISE ( $\mathrm{Li}$ et al. 2017a) and circRNAwrap (Li et al. 2019),

242 considered the circRNAs predicted by the union or the intersection of all methods, respectively,

243 without validating which method combination was best. Our data show that those two approaches

244 could be suboptimal as they suffer either low precision or sensitivity, whereas the best tradeoff

245 between precision and recall is achieved with predictions of methods taken pairwise.

246 Recently, large circRNA databases have been compiled using method combination strategies

247 (Vromman et al. 2020). For instance, circAtlas2.0 (Wu et al. 2020) retained circRNAs identified by at

248 least two methods among CIRI2, CIRCexplorer2, Findcirc and DCC; the same combination but

249 replacing DCC with circRNA_finder has been used in CircRic (Ruan et al. 2019). Applying these two

250 approaches to our real-data sets analysis, we observed that they performed better than most of the

251 algorithms except CirComPara2 (Supplementary Table S2), which achieved similar precision but

252 slightly more than 0.2 better recall. Such a result suggests that CirComPara2 could be employed to

253 compile comprehensive and reliable circRNA databases in future works.

254 Importantly, the computational pipelines used to compile circAtlas 2.0 and CircRic were not

255 implemented as software tools available to the scientific community. Instead, we made the automated

256 and computationally efficient CirComPara2 pipeline ready-to-use and portable through a Docker 
container, freeing bioinformaticians from the several difficulties posed by implementing a

258 computational pipeline, such as installation of multiple tools, software portability, code maintenance

259 and documentation (Menegidio et al. 2018).

260 CirComPara2 is the only tool that aggregates various method expression estimates into unified values

261 that eliminate redundant counts of BJR identified by multiple tools without relying on additional re-

262 alignment of the reads. CircRNAwrap, RAISE, and CircAtlas2.0 applied a quantification step

263 downstream of circRNA detection to estimate the circRNA expression. They considered the re-

264 alignment of the reads onto pseudo reference circRNA sequences through Sailfish-cir (Li et al.

265 2017b), RAISE itself, and CIRIquant (Zhang et al. 2020), respectively, thus increasing the

266 computational requirements of the pipeline. Nevertheless, both Sailfish-cir and CIRIquant can be

267 easily applied downstream to CirComPara2 predictions.

268 CircRNA studies are likely to grow in several branches of biology, both on model (Weigelt et al.

269 2020) and non-model organisms and beyond the biomedical field (Wu et al. 2021a; Chu et al. 2021;

270 Liang et al. 2019), prompting the development of improved tools allowing more extensive circRNA

271 investigation to unravel circRNA-related condition peculiarities, such as differential circRNA

272 expression (Gaffo et al. 2019; Wu et al. 2021c; Tian et al. 2020), imbalances of the CLP (Buratin et

273 al. 2020), and prevailing circular transcript isoform expression (Izuogu et al. 2018). CirComPara2 is a

274 resource tool meeting these needs, as already proved by the successful application of its embryonic

275 implementations in several studies of human diseases and of other species, including plants (Gaffo et

276 al. 2019; Frydrych Capelari et al. 2019; Buratin et al. 2020; Dal Molin et al. 2020).

277 In this work, we described the main features of CirComPara2, our automated and computationally

278 efficient software pipeline for circRNA expression characterisation that also allows traditional gene

279 expression analysis and the computation of circRNA to host-gene linear transcript abundance.

280 Importantly, we demonstrated the asset given by the CirComPara2 method combination strategy for

281 circRNA discovery, which provides robust and inclusive predictions in diverse biological contexts.

282 With CirComPara2, we aim to provide a helpful bioinformatics tool to obtain a more comprehensive 
283 picture of transcriptomes and boost the understanding of circRNA features, biological and

284 pathogenetic roles.

\section{Methods}

\section{Simulated data set}

287 CircRNA reads were simulated with the CIRI_simulator from the CIRI2 tool suite using the whole

288 GRCh38 human genome and Gencode v29 gene annotation.

289 The parameters used in ciri_simulator were: -C 20 -LC 0 -R 1 -LR 1 -L 101 -E 1 -CHR1 0 -M 250 -

290 M2 450 -PM 0 -S 70 -S2 0 -SE 0 -PSI 10.

291 A total of $10 \%$ (30\% for the highly pruned annotation simulation) of the gene annotation for the

292 simulated circRNA parent genes was removed from the annotation file and used as input to circRNA

293 detection methods and to simulate linear transcript reads with Polyester (the reads per_transcript

294 parameter was set to 300). The linear transcript read files were then concatenated to the circRNA read

295 files.

296 Code for generating the simulated data is available as a software tool CCP_simulator at

297 https://github.com/egaffo/CCP_simulator. The parameter ANNOPARTS used in CCP_simulator for

298 the two filters on gene annotation (standard and high pruning) was set to " $85,4,5,1,0,0,0 "$ and

299 “60,15,10,2.5,6,3.5,3”, respectively.

300 Real-data sets

301 Overall, 71 samples with matched rRNA depletion and rRNA depletion followed by RNase R 302 treatment libraries from six studies (Table 1), for a total of 142 samples processed, were retrieved 303 from Gene Expression Omnibus (GEO) or the National Genomics Data Center (NGDC) databases.

304 Reads from PRJCA000751 were trimmed to $150 \mathrm{bp}$ in the preprocessing phase, as reported in the 305 original work.

306 Method predictions in genomic scaffolds or from the mitochondrial genome were not considered.

307 CircRNAs predicted with a length shorter than the library read length or longer than the longest gene 308 expressed in the sample (computed as genes with TPM $\geq 1$ computed by StringTie v2.1.4) were 
filtered out.

310

311

312

313

314

315

316

317

318

319

320

321

\section{CircRNA detection methods' parameters}

The following genome and gene annotations from the Ensembl database were used in the analyses: GRCh38 human genome and v97 gene annotation, Mmul 10 Macaca mulatta genome and v101 gene annotation, and GRCm38 Mus musculus genome and v101 gene annotation.

CirComPara2 default parameters were set for the analyses, which are as follows:

adaptors from the Trimmomatic v0.39 TruSeq3-PE-2.fa file; PREPROCESSOR = "trimmomatic"; PREPROCESSOR_PARAMS $=$ "MAXINFO:40:0.5 LEADING:20 TRAILING:20 SLIDINGWINDOW:4:30 MINLEN:50 AVGQUAL:26". For the PRJCA000751 data sets, the CROP:150 option was appended to the parameter. STAR_PARAMS = '--runRNGseed 123 -outSJfilterOverhangMin $15 \quad 15 \quad 15 \quad 15$--alignSJoverhangMin 15 --alignSJDBoverhangMin 15 -seedSearchStartLmax 30 --outFilterScoreMin 1 --outFilterMatchNmin 1 --outFilterMismatchNmax 2 --chimSegmentMin 15 --chimScoreMin 15 --chimScoreSeparation 10 --chimJunctionOverhangMin 15'. CIRCRNA_METHODS = 'circexplorer2_bwa, circexplorer2_segemeh1, circexplorer2_star, circexplorer2_tophat, ciri, dcc, findcirc' ('circrna_finder' and 'testrealign' values were used in additional runs to obtain CircRNA_finder and Segemhel predictions); CPUS =12; BWA_PARAMS = '-T 19'; SEGEMEHL_PARAMS = '-D 0'; BOWTIE2_PARAMS = '--reorder --score-min=C,-15,0 -q --seed 123'; DCC_EXTRA_PARAMS = '-fg -M -F -Nr 11 -N'; TESTREALIGN_PARAMS = 'q median_1'; FINDCIRC_EXTRA_PARAMS = '--best-qual 40 --filter-tags UNAMBIGUOUS_BP -filter-tags ANCHOR_UNIQUE' (this setting implements the optimization suggested by Hansen (Hansen 2018)). MIN_METHODS $=2 ;$ MIN_READS $=2 ;$ CIRC_MAPPING = “ $\{$ 'SE': ['STAR','TOPHAT','BOWTIE2'],'PE':['BWA','SEGEMEHL']\}"; HISAT2_PARAMS = '--seed 123'.

Segemehl predictions reported in the sngl.bed and trns.txt files were merged to include spliced reads spanning $>20,000$ bps, as CirComPara2 performs it.

Read alignment and circRNA detection methods' parameters were set as the value in the corresponding CirComPara2 parameters. All other parameters not mentioned were left with default values. 


\section{Details of the CirComPara2 method}

337 CircRNA expression estimates in CirComPara2 are represented as the sum of all unique back-splice

338 junction reads (BJRs) identified by the circRNA methods. Then, the number of BJR fragments is

339 counted while keeping track of the number of methods detecting each circRNAs. Finally, circRNAs

340 with at least two reads identified by two or more methods are reported. The MIN_METHODS and

341 MIN_READS options can be used to modify the required minimum reads and methods.

342 The CirComPara2 pipeline considers a preliminary alignment step that maps the reads linearly on the

343 genome. Linearly aligned reads are then used to characterise canonical gene and transcript expression

344 and count the linearly spliced reads spanning the back-splice junctions. Instead, linearly unmapped

345 reads are used as input to the circRNA detection methods.

\section{Evaluation metrics and statistical tests}

347 In evaluating method predictions, to compare samples with their matched control libraries, the 348 different sequencing depths of the control library and the biochemical variability of the 349 exoribonuclease in RNase R treatment have been adjusted by considering the proportion between the expression of the predicted circRNAs and the linear transcripts sharing the back-spliced exons. In

351 each sample and for each method, we computed the circular-to-linear expression proportion (CLP) of 352 each predicted circRNA by counting the number of reads back-spliced $\left(\mathrm{BS}_{\text {reads }}\right)$ and linearly-spliced 353 (LS $\left.\mathrm{S}_{\text {reads }}\right)$ on the circRNA back-splice junctions, respectively. Then, the CLPs were calculated as $354 \mathrm{BS}_{\text {reads }}\left(\mathrm{BS}_{\text {reads }}+\mathrm{LS}_{\text {reads }}\right)$. Szabo and Salzman (Szabo and Salzman 2016) also suggested using the ratio 355 between the expression of circRNAs and their linear counterparts to overcome the evaluation issues 356 deriving by RNase R treated control samples.

357 Assuming that RNase $\mathrm{R}$ should degrade linear transcripts more than circRNAs regardless of its 358 efficiency in different samples, circRNAs with an equal or increased CLP in the control samples were 359 deemed true-positives (TPs); false-positives (FPs) otherwise. Only circRNAs detected in the control 360 libraries were considered to limit incorrect FP calls due to a lower sequencing depth of the control 361 sample and to RNase R sensitive circRNAs (Jeck et al. 2013).

362 The precision was defined as $\mathrm{TP} /(\mathrm{TP}+\mathrm{FP})$. The recall was computed as $\mathrm{TP} /(\mathrm{TP}+\mathrm{FN})$ and $\mathrm{F}_{1}$-score 
as $(2 \times$ Precision $\times$ Recall $) /($ Precision + Recall $)$, where TP and FN denote the true-positive and false-

364 negative numbers.

365 Wilcoxon one-tailed paired tests were used to compare CirComPara2 greater recall and $\mathrm{F}_{1}$-score or

366 lower precision with each method. Bonferroni multiple test correction was applied to compute

367 adjusted $\mathrm{p}$-values (q-values). The q-values reported in the main text refer to the highest value among

368 the pairwise comparisons for recall and $F_{1}$-score, whereas to the lowest value for precision

369 comparisons.

\section{Software versions}

371 The software version of the circRNA detection methods and chimeric read aligners used in this study:

372 CIRCexplorer2 (Zhang et al. 2016) v2.3.8, CircRNA_finder (Westholm et al. 2014) v1.1, CIRI2 (Gao

373 et al. 2018) v2.0.6, DCC v0.4.8 (Cheng et al. 2016), Findcirc (Memczak et al. 2013) v1.2, Segemehl

374 (Hoffmann et al. 2014) v0.3.4 (used also by CIRCexplorer2), BWA MEM (Li 2013) v0.7.15 (used by

375 CIRI2 and CIRCexplorer2), STAR (Dobin et al. 2013) v2.6.1e (used by circRNA_finder, DCC, and

376 CIRCexplorer2), TopHat2 (Kim and Salzberg 2011) v2.1.0 (used for the TopHat-Fusion algorithm by

377 CIRCexplorer2). CirComPara2 v0.1.2.1 was used to run the analysis.

\section{Data access}

379 All the analysed data have been previously published and the accessions are referenced in Table 1.

\section{Competing interests}

381 The authors declare that they have no competing interests.

\section{Acknowledgements}

383 We thank Dr Geertruij te Kronnie for insightful suggestions and critical revision of the manuscript.

\section{Funding}

385 This work has been supported by the Italian Ministry of Education, Universities, and Research grant 386 PRIN 2017 2017PPS2X4_003 (S.B.), AIRC, Milan, Italy Investigator Grant 201720052 (S.B.), and 387 Fondazione Umberto Veronesi, Milan, Italy Fellowship 2020 (E.G.). 
388

389

390

391

392

393

394

395

396

397

398

399

400

401

402

403

404

405

406

407

408

409

410

411

\section{Author Contributions}

Conceptualisation, E.G.; Data curation, E.G.; Formal Analysis, E.G. and A.B.; Funding Acquisition, E.G. and S.B.; Methodology, E.G. and A.B.; Project Administration, S.B.; Resources, S.B.; Software, E.G. and A.B.; Supervision, E.G. and S.B.; Visualization, E.G.; Writing - Original Draft, E.G., A.B. and S.B.; Writing - Review \& Editing, E.G., A.B., A.D.M. and S.B. All authors read and approved the final manuscript.

\section{References}

Andrews S. 2010. FastQC: a quality control tool for high throughput sequence data. FastQC. http://www.bioinformatics.babraham.ac.uk/projects/fastqc (Accessed May 5, 2021).

Awan FM, Yang BB, Naz A, Hanif A, Ikram A, Obaid A, Malik A, Janjua HA, Ali A, Sharif S. 2021. The emerging role and significance of circular RNAs in viral infections and antiviral immune responses: possible implication as theranostic agents. RNA Biol 18: 1-15.

Barrett T, Wilhite SE, Ledoux P, Evangelista C, Kim IF, Tomashevsky M, Marshall KA, Phillippy KH, Sherman PM, Holko M, et al. 2013. NCBI GEO: archive for functional genomics data sets-update. Nucleic Acids Res 41: D991-5.

Bolger AM, Lohse M, Usadel B. 2014. Trimmomatic: a flexible trimmer for Illumina sequence data. Bioinformatics 30: 2114-2120. http://dx.doi.org/10.1093/bioinformatics/btu170.

Bonizzato A, Gaffo E, Te Kronnie G, Bortoluzzi S. 2016. CircRNAs in hematopoiesis and hematological malignancies. Blood Cancer J 6: e483.

Buratin A, Paganin M, Gaffo E, Dal Molin A, Roels J, Germano G, Siddi MT, Serafin V, De Decker M, Gachet S, et al. 2020. Large-scale circular RNA deregulation in T-ALL: unlocking unique ectopic expression of molecular subtypes. Blood Adv 4: 5902-5914.

Cheng J, Metge F, Dieterich C. 2016. Specific identification and quantification of circular RNAs from sequencing data. Bioinformatics 32: 1094-1096. 
Chen I, Chen C-Y, Chuang T-J. 2015. Biogenesis, identification, and function of exonic circular RNAs. Wiley Interdiscip Rev RNA 6: 563-579.

Chen L, Wang C, Sun H, Wang J, Liang Y, Wang Y, Wong G. 2020. The bioinformatics toolbox for circRNA discovery and analysis. Brief Bioinform. http://dx.doi.org/10.1093/bib/bbaa001.

Chen S, Huang V, Xu X, Livingstone J, Soares F, Jeon J, Zeng Y, Hua JT, Petricca J, Guo H, et al. 2019. Widespread and Functional RNA Circularization in Localized Prostate Cancer. Cell 176: 831-843.e22.

Chen Y, Lun ATL, Smyth GK. 2016. From reads to genes to pathways: differential expression analysis of RNA-Seq experiments using Rsubread and the edgeR quasi-likelihood pipeline. F1000Res 5: 1438.

Chu Q, Zheng W, Su H, Zhang L, Chang R, Gao W, Xu T. 2021. A Highly Conserved Circular RNA circRasGEF1B Enhances Antiviral Immunity by Regulating miR-21-3p/MITA Pathway in Lower Vertebrates. $J$ Virol. http://dx.doi.org/10.1128/JVI.02145-20.

Dal Molin A, Hofmans M, Gaffo E, Buratin A, Cavé H, Flotho C, de Haas V, Niemeyer CM, Stary J, Van Vlierberghe P, et al. 2020. CircRNAs Dysregulated in Juvenile Myelomonocytic Leukemia: CircMCTP1 Stands Out. Front Cell Dev Biol 8: 613540.

Dobin A, Davis CA, Schlesinger F, Drenkow J, Zaleski C, Jha S, Batut P, Chaisson M, Gingeras TR. 2013. STAR: ultrafast universal RNA-seq aligner. Bioinformatics 29: 15-21.

Du WW, Yang W, Liu E, Yang Z, Dhaliwal P, Yang BB. 2016. Foxo3 circular RNA retards cell cycle progression via forming ternary complexes with p21 and CDK2. Nucleic Acids Res 44: 28462858 .

Frydrych Capelari É, da Fonseca GC, Guzman F, Margis R. 2019. Circular and Micro RNAs from Flowers Are Simultaneously Isolated from AGO-IP Libraries. Plants 8. http://dx.doi.org/10.3390/plants8090302. 
Gaffo E, Boldrin E, Dal Molin A, Bresolin S, Bonizzato A, Trentin L, Frasson C, Debatin K-M, Meyer LH, Te Kronnie G, et al. 2019. Circular RNA differential expression in blood cell populations and exploration of circRNA deregulation in pediatric acute lymphoblastic leukemia. Sci Rep 9: 14670.

Gaffo E, Bonizzato A, Kronnie GT, Bortoluzzi S. 2017. CirComPara: A Multi-Method Comparative Bioinformatics Pipeline to Detect and Study circRNAs from RNA-seq Data. Noncoding RNA 3. http://dx.doi.org/10.3390/ncrna3010008.

Gao Y, Wang J, Zheng Y, Zhang J, Chen S, Zhao F. 2016. Comprehensive identification of internal structure and alternative splicing events in circular RNAs. Nat Commun 7: 12060.

Gao Y, Zhang J, Zhao F. 2018. Circular RNA identification based on multiple seed matching. Brief Bioinform 19: 803-810.

Hanniford D, Ulloa-Morales A, Karz A, Berzoti-Coelho MG, Moubarak RS, Sánchez-Sendra B, Kloetgen A, Davalos V, Imig J, Wu P, et al. 2020. Epigenetic Silencing of CDR1as Drives IGF2BP3-Mediated Melanoma Invasion and Metastasis. Cancer Cell 37: 55-70.e15.

Hansen TB. 2018. Improved circRNA Identification by Combining Prediction Algorithms. Front Cell Dev Biol 6: 20.

Hansen TB, Jensen TI, Clausen BH, Bramsen JB, Finsen B, Damgaard CK, Kjems J. 2013. Natural RNA circles function as efficient microRNA sponges. Nature 495: 384-388.

Hansen TB, Venø MT, Damgaard CK, Kjems J. 2016. Comparison of circular RNA prediction tools. Nucleic Acids Res 44: e58.

Hoffmann S, Otto C, Doose G, Tanzer A, Langenberger D, Christ S, Kunz M, Holdt LM, Teupser D, Hackermüller J, et al. 2014. A multi-split mapping algorithm for circular RNA, splicing, transsplicing and fusion detection. Genome Biol 15: R34.

Hua JT, Chen S, He HH. 2019. Landscape of Noncoding RNA in Prostate Cancer. Trends Genet 35: 
840-851.

461

462

463

464

465

466

467

468

469

470

471

472

473

474

475

476

477

478

479

480

481

482

Izuogu OG, Alhasan AA, Mellough C, Collin J, Gallon R, Hyslop J, Mastrorosa FK, Ehrmann I, Lako M, Elliott DJ, et al. 2018. Analysis of human ES cell differentiation establishes that the dominant isoforms of the lncRNAs RMST and FIRRE are circular. BMC Genomics 19: 276.

Jeck WR, Sorrentino JA, Wang K, Slevin MK, Burd CE, Liu J, Marzluff WF, Sharpless NE. 2013. Circular RNAs are abundant, conserved, and associated with ALU repeats. RNA 19: 141-157.

Ji P, Wu W, Chen S, Zheng Y, Zhou L, Zhang J, Cheng H, Yan J, Zhang S, Yang P, et al. 2019. Expanded Expression Landscape and Prioritization of Circular RNAs in Mammals. Cell Rep 26: 3444-3460.e5.

Kim D, Paggi JM, Park C, Bennett C, Salzberg SL. 2019. Graph-based genome alignment and genotyping with HISAT2 and HISAT-genotype. Nat Biotechnol 37: 907-915.

Kim D, Salzberg SL. 2011. TopHat-Fusion: an algorithm for discovery of novel fusion transcripts. Genome Biol 12: R72.

Langmead B, Salzberg SL. 2012. Fast gapped-read alignment with Bowtie 2. Nat Methods 9: 357359.

Liang Y, Zhang Y, Xu L, Zhou D, Jin Z, Zhou H, Lin S, Cao J, Huang L. 2019. CircRNA Expression Pattern and ceRNA and miRNA-mRNA Networks Involved in Anther Development in the CMS Line of Brassica campestris. International Journal of Molecular Sciences 20: 4808. http://dx.doi.org/10.3390/ijms20194808.

Li H. 2013. Aligning sequence reads, clone sequences and assembly contigs with BWA-MEM. http://arxiv.org/abs/1303.3997 (Accessed October 7, 2020).

Li L, Bu D, Zhao Y. 2019. CircRNAwrap - a flexible pipeline for circRNA identification, transcript prediction, and abundance estimation. FEBS Lett 593: 1179-1189. 
483

484

485

486

487

488

489

490

491

492

493

494

495

496

497

498

499

500

501

502

503

504

505

506

Li L, Zheng Y-C, Kayani MUR, Xu W, Wang G-Q, Sun P, Ao N, Zhang L-N, Gu Z-Q, Wu L-C, et al. 2017a. Comprehensive analysis of circRNA expression profiles in humans by RAISE. Int $J$ Oncol 51: 1625-1638.

Li M, Xie X, Zhou J, Sheng M, Yin X, Ko E-A, Zhou T, Gu W. 2017b. Quantifying circular RNA expression from RNA-seq data using model-based framework. Bioinformatics 33: 2131-2139.

Li Z, Huang C, Bao C, Chen L, Lin M, Wang X, Zhong G, Yu B, Hu W, Dai L, et al. 2015. Exonintron circular RNAs regulate transcription in the nucleus. Nat Struct Mol Biol 22: 256-264.

Love MI, Soneson C, Hickey PF, Johnson LK, Pierce NT, Shepherd L, Morgan M, Patro R. 2020. Tximeta: Reference sequence checksums for provenance identification in RNA-seq. PLoS Comput Biol 16: e1007664.

Memczak S, Jens M, Elefsinioti A, Torti F, Krueger J, Rybak A, Maier L, Mackowiak SD, Gregersen LH, Munschauer M, et al. 2013. Circular RNAs are a large class of animal RNAs with regulatory potency. Nature 495: 333-338.

Menegidio FB, Jabes DL, Costa de Oliveira R, Nunes LR. 2018. Dugong: a Docker image, based on Ubuntu Linux, focused on reproducibility and replicability for bioinformatics analyses. Bioinformatics 34: 514-515.

National Genomics Data Center Members and Partners. 2020. Database Resources of the National Genomics Data Center in 2020. Nucleic Acids Res 48: D24-D33.

Pertea M, Pertea GM, Antonescu CM, Chang T-C, Mendell JT, Salzberg SL. 2015. StringTie enables improved reconstruction of a transcriptome from RNA-seq reads. Nature Biotechnology 33: 290295. http://dx.doi.org/10.1038/nbt.3122.

Quinlan AR, Hall IM. 2010. BEDTools: a flexible suite of utilities for comparing genomic features. Bioinformatics 26: 841-842. http://dx.doi.org/10.1093/bioinformatics/btq033.

Rajappa A, Banerjee S, Sharma V, Khandelia P. 2020. Circular RNAs: Emerging Role in Cancer 

Med 11: 55. Therapeutic Perspective. Mol Ther 27: 1350-1363.

Slack FJ, Chinnaiyan AM. 2019. The Role of Non-coding RNAs in Oncology. Cell 179: 1033-1055. http://dx.doi.org/10.1016/j.cell.2019.10.017. estimates improve gene-level inferences. F1000Res 4: 1521.

Szabo L, Salzman J. 2016. Detecting circular RNAs: bioinformatic and experimental challenges. Nature Reviews Genetics 17: 679-692. http://dx.doi.org/10.1038/nrg.2016.114. April 23, 2021). and Bioinformatics Analysis of CircRNA in PDGF-BB-Induced Vascular Smooth Muscle Cells. Front Genet 11: 530. circular RNA databases. Brief Bioinform. http://dx.doi.org/10.1093/bib/bbz175. 279.e5.

Westholm JO, Miura P, Olson S, Shenker S, Joseph B, Sanfilippo P, Celniker SE, Graveley BR, Lai 

sequence properties and age-dependent neural accumulation. Cell Rep 9: 1966-1980.

532

533

Wu Q, Ning X, Sun L. 2021a. Megalocytivirus Induces Complicated Fish Immune Response at Multiple RNA Levels Involving mRNA, miRNA, and circRNA. Int J Mol Sci 22. http://dx.doi.org/10.3390/ijms22063156.

Wu W, Ji P, Zhao F. 2020. CircAtlas: an integrated resource of one million highly accurate circular RNAs from 1070 vertebrate transcriptomes. Genome Biol 21: 101.

Wu X, Xiao S, Zhang M, Yang L, Zhong J, Li B, Li F, Xia X, Li X, Zhou H, et al. 2021b. A novel protein encoded by circular SMO RNA is essential for Hedgehog signaling activation and glioblastoma tumorigenicity. Genome Biol 22: 33.

Wu Y, Zhao T, Deng R, Xia X, Li B, Wang X. 2021c. A study of differential circRNA and lncRNA expressions in COVID-19-infected peripheral blood. Sci Rep 11: 7991.

Xiao M-S, Ai Y, Wilusz JE. 2020. Biogenesis and Functions of Circular RNAs Come into Focus. Trends Cell Biol 30: 226-240.

Xiao M-S, Wilusz JE. 2019. An improved method for circular RNA purification using RNase R that efficiently removes linear RNAs containing G-quadruplexes or structured 3' ends. Nucleic Acids Res 47: 8755-8769.

Zeng X, Lin W, Guo M, Zou Q. 2017. A comprehensive overview and evaluation of circular RNA detection tools. PLoS Comput Biol 13: e1005420.

Zhang J, Chen S, Yang J, Zhao F. 2020. Accurate quantification of circular RNAs identifies extensive circular isoform switching events. Nat Commun 11: 90.

Zhang X-O, Dong R, Zhang Y, Zhang J-L, Luo Z, Zhang J, Chen L-L, Yang L. 2016. Diverse alternative back-splicing and alternative splicing landscape of circular RNAs. Genome Res 26: $1277-1287$. 
bioRxiv preprint doi: https://doi.org/10.1101/2021.02 18.431705; this version posted May 21, 2021. The copyright holder for this preprint

(which was not certified by peer review) is the author/funder, who has granted bioRxiv a license to display the preprint in perpetuity. It is made available under aCC-BY-NC-ND 4.0 International license.

554 Zhang X-O, Wang H-B, Zhang Y, Lu X, Chen L-L, Yang L. 2014. Complementary sequence-

$555 \quad$ mediated exon circularization. Cell 159: 134-147.

556 Zhu A, Ibrahim JG, Love MI. 2019. Heavy-tailed prior distributions for sequence count data: removing the noise and preserving large differences. Bioinformatics 35: 2084-2092. 